TAIWANESE JOURNAL OF MATHEMATICS

Vol. 13, No. 2A, pp. 545-558, April 2009

This paper is available online at http://www.tjm.nsysu.edu.tw/

\title{
ON GLOBAL SOLUTIONS AND BLOW-UP OF SOLUTIONS FOR A NONLINEARLY DAMPED PETROVSKY SYSTEM
}

\author{
Shun-Tang Wu and Long-Yi Tsai
}

\begin{abstract}
We consider the initial boundary value problem for a Petrovsky system with nonlinear damping

$$
u_{t t}+\Delta^{2} u+a\left|u_{t}\right|^{m-2} u_{t}=b|u|^{p-2} u,
$$

in a bounded domain. We showed that the solution is global in time under some conditions without the relation between $m$ and $p$. We also prove that the local solution blows-up in finite time if $p>m$ and the initial energy is nonngeative. The decay estimates of the energy function and the estimates of the lifespan of solutions are given. In this way, we can extend the result of ([6]).
\end{abstract}

\section{INTRODUCTION}

In this paper we are concerned with the initial boundary value problem for the following Petrovsky equation :

$$
u_{t t}+\Delta^{2} u+a\left|u_{t}\right|^{m-2} u_{t}=b|u|^{p-2} u
$$

with initial conditions

$$
u(x, 0)=u_{0}(x), u_{t}(x, 0)=u_{1}(x), x \in \Omega,
$$

and boundary condition

$$
u(x, t)=\frac{\partial u}{\partial \nu}(x, t)=0, x \in \partial \Omega, t \geq 0,
$$

Accepted February 25, 2007.

Communicated by Chiun-Chuan Chen.

2000 Mathematics Subject Classification: 35G10, 35Q72.

Key words and phrases: Global, Blow-up, Life span, Nonlinear damping, Petrovsky system. 
where $\Omega \subset R^{N}, N \geq 1$, is a bounded domain with a smooth boundary $\partial \Omega$ so that Divergence theorem can be applied and $\nu$ be the unit normal vector pointing toward the exterior of $\Omega$ and let $\frac{\partial}{\partial \nu}$ denotes the normal derivative and $a, b$ are some positive constants and $p, m>2$.

Guesmia ([2]) studied the problem

$$
u_{t t}+\Delta^{2} u+q(x) u+g\left(u_{t}\right)=0,
$$

where $q: \Omega \rightarrow R^{+}$is a bounded function. Under some assumptions, he showed the solution of (1.4) decays exponentially if $g$ behaves like a linear function, whereas the decay is ploynomially otherwise. Later, Guesmia ([3]) concerned equation (1.4) coupled with a semilinear wave equation and derived similar results. In the related problems, we can cite $([4,5,8])$ and the references therein. Recently, Messaoudi ([6]) investigated problem (1.1) and showed the solution blows-up in finite time if $p>m$ in the case that the initial energy is negative. On the other hand, he also proved the solution is global in time if $m \geq p$. However, no decay rate of the global solution is given and no blow-up result is discussed for the initial energy being nonnegative.

In this paper we shall prove the global existence result without the relation between $m$ and $p$ and show that the energy function decays algebraically under some conditions. On the other hand, we also establish the blow-up properties of local solution for problem $(1.1)-(1.3)$ with nonpositive initial energy as well as small positive initial energy. In this way, we can extend the result of ([6]). The content of this paper is organized as follows. In section 2, we give some lemmas and the local existence theorem 2.3 in ([6]). In section 3, we define an energy function $E(t)$ in (3.3) and show that it is a nonincreasing function of $t$. We obtain global existence and decay properties of the solutions of $(1.1)-(1.3)$ which are given in Theorem 3.5. Finally, the blow-up properties of $(1.1)-(1.3)$ and the estimates for the blow-up time $T^{*}$ are also given in the last section.

\section{Preliminary Results}

In this section, we shall give some lemmas which will be used throughout this work.

Lemma 2.1. (Sobolev-Poincare inequality)([1]) If $1 \leq p \leq \frac{2 N}{[N-2 m]^{+}}(1 \leq$ $p<\infty$ if $N=2 m$ ), then

$$
\|u\|_{p} \leq B_{1}\left\|(-\Delta)^{\frac{m}{2}} u\right\|_{2}, \text { for } u \in D\left((-\Delta)^{\frac{m}{2}}\right)
$$

holds with some positive constant $B_{1}$, where we put $[a]^{+}=\max \{0, a\}, \frac{1}{[a]^{+}}=\infty$ if $[a]^{+}=0$ and we denote $\|\cdot\|_{p}$ to be the norm of $L^{p}(\Omega)$. 
Lemma 2.2. ([7]) Let $\phi(t)$ be a nonincreasing and nonnegative function on $[0, T], T>1$, such that

$$
\phi(t)^{1+r} \leq \omega_{0}(\phi(t)-\phi(t+1)) \text { on }[0, T],
$$

where $\omega_{0}$ is a positive constant and $r$ is a nonnegative constant. Then we have (i) if $r>0$, then

$$
\phi(t) \leq\left(\phi(0)^{-r}+\omega_{0}^{-1} r[t-1]^{+}\right)^{-\frac{1}{r}} \text { on }[0, T] .
$$

(ii) If $r=0$, then

$$
\phi(t) \leq \phi(0) e^{-\omega_{1}[t-1]^{+}} \text {on }[0, T]
$$

where $\omega_{1}=\ln \left(\frac{\omega_{0}}{\omega_{0}-1}\right)$, here $\omega_{0}>1$.

Next, we state the local existence theorem which is proved in ([6]).

Theorem 2.3. (Local Existence) Suppose that $2<p \leq p^{*}, 2<m \leq$ $m^{*}$ and that $u_{0} \in H_{0}^{2}(\Omega)$ and $u_{1} \in L^{2}(\Omega)$, then there exists a unique solution $u$ of $(1.1)-(1.3)$ satisfying

$$
u \in C\left([0, T] ; H_{0}^{2}(\Omega)\right)
$$

and

$$
u_{t} \in C\left([0, T] ; L^{2}(\Omega)\right) \cap L^{m}(\Omega \times(0, T)) .
$$

Moreover, at least one of the following statements holds true :

(i) $T=\infty$,

$(i i)\left\|u_{t}(t)\right\|_{2}^{2}+\|\Delta u(t)\|_{2}^{2} \rightarrow \infty$ as $t \rightarrow T^{-}$,

where

$$
p^{*}=\frac{2(N-2)}{N-4}(\infty, \text { if } N \leq 4) \text { and } m^{*}=\frac{2 N}{N-4}(\infty, \text { if } N \leq 4) .
$$

\section{Global Existence and Energy Decay}

In this section, we consider the global existence and energy decay of solutions for problem (1.1) - (1.3).

Let

$$
I(t) \equiv I(u(t))=\|\Delta u(t)\|_{2}^{2}-b\|u(t)\|_{p}^{p}
$$

and

$$
J(t) \equiv J(u(t))=\frac{1}{2}\|\Delta u(t)\|_{2}^{2}-\frac{b}{p}\|u(t)\|_{p}^{p}
$$


for $u(t) \in H_{0}^{2}(\Omega), t \geq 0$.We define the energy function of the solution $u$ of $(1.1)-(1.3)$ by

$$
E(t)=\frac{1}{2}\left\|u_{t}\right\|_{2}^{2}+J(t) \text { for } t \geq 0 .
$$

Remark: By (3.3), we have

$$
\begin{aligned}
E(t) & =\frac{1}{2}\left\|u_{t}\right\|_{2}^{2}+\frac{1}{2}\|\Delta u(t)\|_{2}^{2}-\frac{b}{p}\|u\|_{p}^{p} \\
& \geq \frac{1}{2}\|\Delta u(t)\|_{2}^{2}-\frac{b}{p}\|u\|_{p}^{p}, t \geq 0 .
\end{aligned}
$$

By Lemma 2.1, we get

$$
E(t) \geq G\left(\|\Delta u(t)\|_{2}\right), t \geq 0
$$

where

$$
G(\lambda)=\frac{1}{2} \lambda^{2}-\frac{B_{1}^{p} b}{p} \lambda^{p},
$$

here $B_{1}$ is the Sobolev's constant given in Lemma 2.1. Note that $G(\lambda)$ has the maximum at $\lambda_{1}=\left(\frac{1}{b B_{1}^{p}}\right)^{\frac{1}{p-2}}$ and the maximum value is

$$
E_{1}=G\left(\lambda_{1}\right)=b^{-\frac{2}{p-2}}\left(\frac{1}{2}-\frac{1}{p}\right) B_{1}^{\frac{-2 p}{p-2}}
$$

Lemma 3.1. $E(t)$ is a nonincreasing function on $[0, T]$ and

$$
E^{\prime}(t)=-a \int_{\Omega}\left|u_{t}\right|^{m} d x
$$

Proof. By using Divergence theorem and (1.1) - (1.3), we see that (3.7) follows at once.

Lemma 3.2. Assume that $E(0)<E_{1}$. Then

(i) If $\left\|\Delta u_{0}\right\|_{2}<\lambda_{1}$, then $\|\Delta u(t)\|_{2}<\lambda_{1}$ for $t \geq 0$.

(ii) If $\left\|\Delta u_{0}\right\|_{2}>\lambda_{1}$, then there exists $\lambda_{2}>\lambda_{1}$ such that $\|\Delta u(t)\|_{2} \geq \lambda_{2}$ for $t \geq 0$.

Proof. From the definition of $G(\lambda)$, we see that $G(\lambda)$ is increasing in $\left(0, \lambda_{1}\right)$, decreasing in $\left(\lambda_{1}, \infty\right)$ and $G(\lambda) \rightarrow-\infty$ as $\lambda \rightarrow \infty$. Since $E(0)<E_{1}$, so there 
exist $\lambda_{2}^{\prime}$ and $\lambda_{2}$ such that $\lambda_{2}^{\prime}<\lambda_{1}<\lambda_{2}$ and $G\left(\lambda_{2}^{\prime}\right)=G\left(\lambda_{2}\right)=E(0)$. (i) when $\left\|\Delta u_{0}\right\|_{2}<\lambda_{1}$, by (3.5), we have

$$
G\left(\left\|\Delta u_{0}\right\|_{2}\right) \leq E(0)=G\left(\lambda_{2}^{\prime}\right) .
$$

It implies $\left\|\Delta u_{0}\right\|_{2}<\lambda_{2}^{\prime}$.

We claim that $\|\Delta u(t)\|_{2} \leq \lambda_{2}^{\prime}$ for $t>0$. If not, then there exists $t_{0}>0$ such that $\left\|\Delta u\left(t_{0}\right)\right\|_{2}>\lambda_{2}^{\prime}$. Case (a) if $\lambda_{2}^{\prime}<\left\|\Delta u\left(t_{0}\right)\right\|_{2}<\lambda_{2}$, then $G\left(\left\|\Delta u\left(t_{0}\right)\right\|_{2}\right)>$ $E(0) \geq E\left(t_{0}\right)$. It contradicts to (3.5). Case (b) if $\left\|\Delta u\left(t_{0}\right)\right\|_{2} \geq \lambda_{2}$, then by contiunity of $\|\Delta u(t)\|_{2}$, there exists $0<t_{1}<t_{0}$ such that $\lambda_{2}^{\prime}<\left\|\Delta u\left(t_{1}\right)\right\|_{2}<$ $\lambda_{2}$, then $G\left(\left\|\Delta u\left(t_{1}\right)\right\|_{2}\right)>E(0) \geq E\left(t_{1}\right)$. This is a contracdition. (ii) when $\left\|\Delta u_{0}\right\|_{2}>\lambda_{1}$, as in case $(i)$ we also deduce that $\left\|\Delta u_{0}\right\|_{2}>\lambda_{1}$ implies $\|\Delta u(t)\|_{2} \geq$ $\lambda_{2}$ for $t \geq 0$.

Lemma 3.3. Let $u$ be the solution of (1.1) - (1.3). Assume the conditions of Theorem 2.3 hold. If $0<\left\|\Delta u_{0}\right\|_{2}<\lambda_{1}$ and

$$
\alpha=b B_{1}^{p}\left(\frac{2 p}{p-2} E(0)\right)^{\frac{p-2}{2}}<1,
$$

then $I(t)>0$, for $t \in[0, T]$.

Proof. First, we note that $0<\left\|\Delta u_{0}\right\|_{2}<\lambda_{1}$ implies $I(0)>0$, then it follows from the continuity of $u(t)$ that

$$
I(t) \geq 0
$$

for some interval near $t=0$. Let $t_{\max }>0$ be a maximal time (possibly $t_{\max }=T$ ), when (3.9) holds on $\left[0, t_{\max }\right)$. From (3.2) and (3.1), we have

$$
J(t)=\frac{p-2}{2 p}\|\Delta u\|_{2}^{2}+\frac{1}{p} I(t) .
$$

By (3.10), (3.3) and (3.7), we deduce

$$
\|\Delta u\|_{2}^{2} \leq \frac{2 p}{p-2} J(t) \leq \frac{2 p}{p-2} E(t) \leq \frac{2 p}{p-2} E(0) .
$$

Then, by Lemma $2.1,(3.11)$ and (3.8), we obtain

$$
\begin{aligned}
b\|u\|_{p}^{p} & \leq b B_{1}^{p}\|\Delta u\|_{2}^{p} \leq b B_{1}^{p}\left(\frac{2 p}{p-2} E(0)\right)^{\frac{p-2}{2}}\|\Delta u\|_{2}^{2} \\
& =\alpha\|\Delta u\|_{2}^{2}<\|\Delta u(t)\|_{2}^{2} \text { on }\left[0, t_{\max }\right) .
\end{aligned}
$$

Thus

$$
I(t)=\|\Delta u(t)\|_{2}^{2}-b\|u\|_{p}^{p}>0 \text { on }\left[0, t_{\max }\right) .
$$


This implies that we can take $t_{\max }=T$.

Remark. Inequality (3.8) (i.e. $\alpha<1$ ) is equivalent to $E(0)<E_{1}$.

Lemma 3.4. Let $u$ satisfies the assumptions of Lemma 3.3. Then there exists $0<\eta<1$ such that

$$
b\|u(t)\|_{p}^{p} \leq(1-\eta)\|\Delta u(t)\|_{2}^{2} \text { on }[0, T],
$$

where $\eta=1-\alpha$.

Proof. From (3.12), we get

$$
b\|u(t)\|_{p}^{p} \leq \alpha\|\Delta u(t)\|_{2}^{2}, t \in[0, T] .
$$

Let $\eta=1-\alpha$, then we have the result.

Remark. From Lemma 3.4, we can deduce that

$$
\|\Delta u(t)\|_{2}^{2} \leq \frac{1}{\eta} I(t), t \in[0, T]
$$

Theorem 3.5. ([Global Existence and Energy decay]). Suppose that $0<$ $\left\|\Delta u_{0}\right\|_{2}<\lambda_{1}$ and $0<E(0)<E_{1}$, then the problem (1.1) - (1.3) admits a global solution $u$ if $u_{0} \in H_{0}^{2}(\Omega)$ and $u_{1} \in L^{2}(\Omega)$. Furthermore, we have the following decay estimates:

$$
E(t) \leq\left(E(0)^{-\frac{m-2}{2}}+\frac{(m-2) \tau}{2}[t-1]^{+}\right)^{-\frac{2}{m-2}} \text { on }[0, \infty),
$$

where $\tau$ is given in (3.27).

Proof. First, we want to show that the solution of $(1.1)-(1.3)$ is global in time in the sense of Theorem 2.3. From (3.3) and (3.11), we see that

$$
\left\|u_{t}\right\|_{2}^{2}+\|\Delta u\|_{2}^{2} \leq\left(2+\frac{2 p}{p-2}\right) E(0) .
$$

Then, by Theorem 2.3, we have the global existence result. By integrating (3.7) over $[t, t+1], t>0$, we have

$$
E(t)-E(t+1) \equiv D(t)^{m}
$$

where

$$
D(t)^{m}=a \int_{t}^{t+1}\left\|u_{t}\right\|_{m}^{m} d t
$$


By virture of (3.15) and Hölder inequality, we observe that

$$
\int_{t}^{t+1} \int_{\Omega}\left|u_{t}\right|^{2} d x d t \leq c(\Omega) D(t)^{2}
$$

where $c(\Omega)=(\operatorname{vol}(\Omega))^{\frac{m-2}{m}}$. Hence, from (3.16), there exist $t_{1} \in\left[t, t+\frac{1}{4}\right]$ and $t_{2} \in\left[t+\frac{3}{4}, t+1\right]$ such that

$$
\left\|u_{t}\left(t_{i}\right)\right\|_{2}^{2} \leq 4 c(\Omega) D(t)^{2}, \quad i=1,2
$$

Next, multiplying (1.1) by $u$ and integrating it over $\Omega \times\left[t_{1}, t_{2}\right]$, we get

$$
\int_{t_{1}}^{t_{2}} I(t) d t=-\int_{t_{1}}^{t_{2}} \int_{\Omega} u_{t t} u d x d t-a \int_{t_{1}}^{t_{2}} \int_{\Omega}\left|u_{t}\right|^{m-2} u_{t} u d x d t
$$

Then, by using (1.1) and integrating by parts on the first term of the right hand side of (3.18), we obtain

$$
\begin{aligned}
& \int_{t_{1}}^{t_{2}} I(t) d t \\
& \leq \sum_{i=1}^{2}\left\|u_{t}\left(t_{i}\right)\right\|_{2}\left\|u\left(t_{i}\right)\right\|_{2}+\int_{t_{1}}^{t_{2}}\left\|u_{t}\right\|_{2}^{2} d t-a \int_{t_{1}}^{t_{2}} \int_{\Omega}\left|u_{t}\right|^{m-2} u_{t} u d x d t
\end{aligned}
$$

By Hölder inequality and Poincaré inequality, it follows that

$$
\begin{aligned}
& \left.\left|a \int_{t_{1}}^{t_{2}} \int_{\Omega}\right| u_{t}\right|^{m-2} u_{t} u d x d t \leq a \int_{t_{1}}^{t_{2}}\|u\|_{m}\left\|u_{t}\right\|_{m}^{m-1} d t \\
\leq & a B_{1} \int_{t_{1}}^{t_{2}}\|\Delta u\|_{2}\left\|u_{t}\right\|_{m}^{m-1} d t \\
\leq & a B_{1}\left(\frac{2 p}{p-2}\right)^{\frac{1}{2}} \sup _{t_{1} \leq s \leq t_{2}} E(s)^{\frac{1}{2}} \int_{t_{1}}^{t_{2}}\left\|u_{t}\right\|_{m}^{m-1} d t \\
\leq & a B_{1}\left(\frac{2 p}{p-2}\right)^{\frac{1}{2}} D(t)^{m-1} \sup _{t_{1} \leq s \leq t_{2}} E(s)^{\frac{1}{2}} .
\end{aligned}
$$

And by using (3.17), Poincaré inequality and (3.11), we also have

$$
\left\|u_{t}\left(t_{i}\right)\right\|_{2}\left\|u\left(t_{i}\right)\right\|_{2} \leq c_{1} D(t) \sup _{t_{1} \leq s \leq t_{2}} E(s)^{\frac{1}{2}}
$$


where $c_{1}=2 B_{1} \sqrt{c(\Omega)}\left(\frac{2 p}{p-2}\right)^{\frac{1}{2}}$. Then by $(3.20)-(3.21)$ and (3.16), we obtain from (3.19)

$$
\begin{aligned}
& \int_{t_{1}}^{t_{2}} I(t) d t \\
\leq & 2 c_{1} D(t) \sup _{t_{1} \leq s \leq t_{2}} E(s)^{\frac{1}{2}}+a B_{1}\left(\frac{2 p}{p-2}\right)^{\frac{1}{2}} D(t)^{m-1} \sup _{t_{1} \leq s \leq t_{2}} E(s)^{\frac{1}{2}} \\
+ & c(\Omega) D(t)^{2} .
\end{aligned}
$$

On the other hand, from (3.3) and using (3.10) and (3.13), we deduce

$$
E(t) \leq \frac{1}{2}\left\|u_{t}\right\|_{2}^{2}+c_{2} I(t)
$$

where $c_{2}=\left(\frac{p-2}{2 p \eta}+\frac{1}{p}\right)$. By integrating (3.23) over $\left(t_{1}, t_{2}\right)$, we obtain

$$
\int_{t_{1}}^{t_{2}} E(t) d t \leq \frac{1}{2} \int_{t_{1}}^{t_{2}}\left\|u_{t}\right\|_{2}^{2} d t+c_{2} \int_{t_{1}}^{t_{2}} I(t) d t
$$

Hence, by (3.16) and (3.22), we have

$$
\begin{aligned}
\int_{t_{1}}^{t_{2}} E(t) d t \leq & \frac{1}{2} c(\Omega) D(t)^{2}+c_{2}\left[2 c_{1} D(t) \sup _{t_{1} \leq s \leq t_{2}} E(s)^{\frac{1}{2}}\right. \\
& \left.+a B_{1}\left(\frac{2 p}{p-2}\right)^{\frac{1}{2}} D(t)^{m-1} \sup _{t_{1} \leq s \leq t_{2}} E(s)^{\frac{1}{2}}+c(\Omega) D(t)^{2}\right] .
\end{aligned}
$$

Moreover, multiplying (1.1) by $u_{t}$ and then integrating it over $\left[t, t_{2}\right] \times \Omega$, we get

$$
E(t)=E\left(t_{2}\right)+a \int_{t}^{t_{2}}\left\|u_{t}\right\|_{m}^{m} d s
$$

Since $t_{2}-t_{1} \geq \frac{1}{2}$, it follows that

$$
E\left(t_{2}\right) \leq 2 \int_{t_{1}}^{t_{2}} E(t) d t
$$

Then, thanks to (3.14), we arrive at

$$
\begin{aligned}
E(t) & \leq 2 \int_{t_{1}}^{t_{2}} E(t) d t+a \int_{t}^{t_{2}}\left\|u_{t}\right\|_{m}^{m} d s \\
& =2 \int_{t_{1}}^{t_{2}} E(t) d t+D(t)^{m} .
\end{aligned}
$$


Thus, by using (3.24) and Lemma 3.2, we see that

$$
\begin{aligned}
E(t) \leq & \left(c(\Omega)+4 c_{1} c_{2}\right) D(t)^{2}+D(t)^{m} \\
& +2 c_{2}\left[2 c_{1} D(t)+a B_{1}\left(\frac{2 p}{p-2}\right)^{\frac{1}{2}} D(t)^{m-1}\right] E(t)^{\frac{1}{2}}, t \geq 0 .
\end{aligned}
$$

Hence, by Young's inequality, we deduce

$$
E(t) \leq c_{3}\left[D(t)^{2}+D(t)^{m}+D(t)^{2(m-1)}\right],
$$

where $c_{3}$ is some positive constant. Therefore, we have the following decay estimate. From (3.26) and (3.14), we get

$$
\begin{aligned}
E(t) & \leq c_{3}\left[1+D(t)^{m-2}+D(t)^{2 m-4}\right] D(t)^{2} \\
& \leq c_{3}\left[1+E(0)^{\frac{m-2}{m}}+E(0)^{\frac{2 m-4}{m}}\right] D(t)^{2} .
\end{aligned}
$$

This implies that

$$
E(t)^{\frac{m}{2}} \leq\left(c_{4}(E(0))\right)^{\frac{m}{2}} D(t)^{m},
$$

where $c_{4}(E(0))=c_{3}\left[1+E(0)^{\frac{m-2}{m}}+E(0)^{\frac{2 m-4}{m}}\right]$. Note that $\lim _{E(0) \rightarrow 0} c_{4}(E(0))=$ $c_{3}>0$. Hence, by applying Lemma 2.2 yields

$$
E(t) \leq\left(E(0)^{-\frac{m-2}{2}}+\frac{(m-2) \tau}{2}[t-1]^{+}\right)^{-\frac{2}{m-2}} \text { on }[0, \infty),
$$

where $\tau=\left(c_{4}(E(0))\right)^{-\frac{m}{2}}$.

\section{BLOW-UP POPERTY}

In this section, we shall show that the solution of problem (1.1) blows up in finite time if $p>m$ and $E(0)<E_{1}$.

Theorem 4.1. (Nonexistence of Global Solutions) Suppose that $p>m$. If one of the following is satisfied

(i) $E(0)<0$

(ii) $0 \leq E(0)<E_{1}$ and $\left\|\Delta u_{0}\right\|_{2}>\lambda_{1}$,

then the local solution of the problem (1.1) - (1.3) blows up at a finite time $T$. The lifespan $T$ is estimated by $0<T \leq \frac{L(0)^{1-\theta}}{c_{11}(\theta-1)}$, here $L(t)$ and $c_{11}$ are given in (4.13) and (4.22) respectively. $\theta$ is a constant given in (4.17). 
Proof. (I) For $0 \leq E(0)<E_{1}$, we set

$$
H(t)=E_{2}-E(t), t \geq 0,
$$

where $E_{2}=\frac{E(0)+E_{1}}{2}$. By (3.7), we see that

$$
H^{\prime}(t)=a \int_{\Omega}\left|u_{t}\right|^{m} d x \geq 0
$$

Thus, we have

$$
H(t) \geq H(0)=E_{2}-E(0)>0, t \geq 0 .
$$

Let

$$
A(t)=\int_{\Omega} u u_{t} d x
$$

By differentiating (4.4) and using (1.1), we obtain

$$
A^{\prime}(t)=\left\|u_{t}\right\|_{2}^{2}-\|\Delta u\|_{2}^{2}-a \int_{\Omega}\left|u_{t}\right|^{m-2} u_{t} u d x+b\|u\|_{p}^{p} .
$$

Hence, by (3.3), we deduce

$$
\begin{aligned}
A^{\prime}(t)= & a_{1}\left\|u_{t}\right\|_{2}^{2}+a_{2}\|\Delta u(t)\|_{2}^{2}-a \int_{\Omega}\left|u_{t}\right|^{m-2} u_{t} u d x \\
& +p H(t)-p E_{2} .
\end{aligned}
$$

where $a_{1}=1+\frac{p}{2}$ and $a_{2}=\frac{p}{2}-1$. We observe that $a_{i}>0, i=1,2$. Moreover

$$
\begin{aligned}
& a_{2}\|\Delta u(t)\|_{2}^{2}-p E_{2} \\
= & a_{2} \frac{\lambda_{2}^{2}-\lambda_{1}^{2}}{\lambda_{2}^{2}}\|\Delta u(t)\|_{2}^{2}+a_{2} \lambda_{1}^{2} \frac{\|\Delta u(t)\|_{2}^{2}}{\lambda_{2}^{2}}-p E_{2} \\
\geq & c_{1}\|\Delta u(t)\|_{2}^{2}+c_{2},
\end{aligned}
$$

where $\lambda_{2}$ is given in Lemma 3.1, $c_{1}=a_{2} \frac{\lambda_{2}^{2}-\lambda_{1}^{2}}{\lambda_{2}^{2}}$ and $c_{2}=a_{2} \lambda_{1}^{2}-p E_{2}$. By Lemma 3.1 (ii), we have $c_{1}>0$ and by (3.6), we see that

$$
\begin{aligned}
c_{2} & =\frac{(p-2) \lambda_{1}^{2}}{2}-\frac{p\left(E_{1}+E(0)\right)}{2} \\
& =\frac{p\left(E_{1}-E(0)\right)}{2}>0 .
\end{aligned}
$$

Thus, by (4.6) and (4.7), we arrive at

$$
A^{\prime}(t) \geq a_{1}\left\|u_{t}\right\|_{2}^{2}+c_{1}\|\Delta u(t)\|_{2}^{2}-a \int_{\Omega}\left|u_{t}\right|^{m-2} u_{t} u d x+p H(t) .
$$


On the other hand, by Hölder inequality, we have

$$
\begin{aligned}
\left.a\left|\int_{\Omega}\right| u_{t}\right|^{m-2} u_{t} u d x \mid & \leq a\|u\|_{m}\left\|u_{t}\right\|_{m}^{m-1} \\
& \leq c_{3}\|u\|_{p}^{1-\frac{p}{m}}\|u\|_{p}^{\frac{p}{m}}\left\|u_{t}\right\|_{m}^{m-1}
\end{aligned}
$$

where $c_{3}=a(\operatorname{vol}(\Omega))^{\frac{p-m}{m p}}$. Note that, from (4.1) and (3.4), we get

$$
\begin{aligned}
H(t) & \leq E_{1}-\frac{1}{2}\|\Delta u\|_{2}^{2}+\frac{b}{p}\|u\|_{p}^{p} \\
& \leq E_{1}-\frac{1}{2} \lambda_{1}^{2}+\frac{b}{p}\|u\|_{p}^{p},
\end{aligned}
$$

where the last inequality is derived by Lemma 3.1(ii). Thus, by (3.6) and (4.3), we see that

$$
0<H(0) \leq H(t) \leq \frac{b}{p}\|u\|_{p}^{p} \text { for } t \geq 0 .
$$

Then, using (4.10), we have from (4.9)

$$
\left.a\left|\int_{\Omega}\right| u_{t}\right|^{m-2} u_{t} u d x \mid \leq c_{4}\|u\|_{p}^{\frac{p}{m}} H(t)^{\frac{1}{p}-\frac{1}{m}}\left\|u_{t}\right\|_{m}^{m-1} .
$$

Hence by Young's inequality and (4.2), we obtain

$$
\left.a\left|\int_{\Omega}\right| u_{t}\right|^{m-2} u_{t} u d x \mid \leq c_{5}\left(\varepsilon^{m}\|u\|_{p}^{p}+\varepsilon^{-m^{\prime}} H^{\prime}(t)\right) H(t)^{-\alpha_{1}}
$$

where $\alpha_{1}=\frac{1}{m}-\frac{1}{p}>0, \varepsilon>0, m^{\prime}=\frac{m}{m-1}, c_{4}=c_{3}\left(\frac{p}{b}\right)^{\frac{1}{p}-\frac{1}{m}}$ and $c_{5}=c_{4} \max \left(1, \frac{1}{a}\right)$. Letting $0<\alpha<\alpha_{1}$ and by (4.10), we see that

$$
\begin{aligned}
& \left.a\left|\int_{\Omega}\right| u_{t}\right|^{m-2} u_{t} u d x \mid \\
\leq & c_{5}\left(\varepsilon^{m} H(0)^{-\alpha_{1}}\|u\|_{p}^{p}+\varepsilon^{-m^{\prime}} H(0)^{\alpha-\alpha_{1}} H(t)^{-\alpha} H^{\prime}(t)\right) .
\end{aligned}
$$

Now, we define

$$
L(t)=H(t)^{1-\alpha}+\delta_{1} A(t), t \geq 0,
$$

where $\delta_{1}$ is a positive constant to be specified later. By differentiating (4.13), and then by (4.12) and (4.8), we see that

$$
\begin{aligned}
L^{\prime}(t) \geq & \left(1-\alpha-\delta_{1} c_{5} \varepsilon^{-m^{\prime}} H(0)^{\alpha-\alpha_{1}}\right) H(t)^{-\alpha} H^{\prime}(t) \\
& +\delta_{1}\left[a_{1}\left\|u_{t}\right\|_{2}^{2}+c_{1}\|\Delta u(t)\|_{2}^{2}+p H(t)\right] \\
& -\delta_{1} c_{5} \varepsilon^{m} H(0)^{-\alpha_{1}}\|u\|_{p}^{p} .
\end{aligned}
$$


Letting $a_{3}=\min \left\{a_{1}, c_{1}, \frac{p}{2}\right\}$ and decomposing $\delta_{1} p H(t)$ in (4.14) by

$$
\delta_{1} p H(t)=2 a_{3} \delta_{1} H(t)+\left(p-2 a_{3}\right) \delta_{1} H(t) .
$$

Thus, by (4.1) and (3.3), we obtain

$$
\begin{aligned}
L^{\prime}(t) \geq( & \left(1-\alpha-\delta_{1} c_{5} \varepsilon^{-m^{\prime}} H(0)^{\alpha-\alpha_{1}}\right) H(t)^{-\alpha} H^{\prime}(t) \\
+ & \delta_{1}\left[\frac{2 a_{3} b}{p}-c_{5} \varepsilon^{m} H(0)^{-\alpha_{1}}\right]\|u\|_{p}^{p}+\delta_{1}\left(a_{1}-a_{3}\right)\left\|u_{t}\right\|_{2}^{2} \\
& \delta_{1}\left(c_{1}-a_{3}\right)\|\Delta u(t)\|_{2}^{2}+\left(p-2 a_{3}\right) \delta_{1} H(t) .
\end{aligned}
$$

Now, we choose $\varepsilon>0$ small such that $\frac{2 a_{3} b}{p}-c_{5} \varepsilon^{m} H(0)^{-\alpha_{1}} \geq \frac{a_{3} b}{2 p}$, and $0<\delta_{1}<$ $\frac{(1-\alpha)}{c_{5}} \varepsilon^{m^{\prime}} H(0)^{\alpha_{1}-\alpha}$. Then (4.15) becomes

$$
L^{\prime}(t) \geq c_{6} \delta_{1}\left(\|u\|_{p}^{p}+\left\|u_{t}\right\|_{2}^{2}+H(t)+\|\Delta u\|_{2}^{2}\right),
$$

here $c_{6}=\min \left\{\frac{a_{3} b}{2 p}, a_{1}-a_{3}, c_{1}-a_{3}, p-2 a_{3}\right\}$. Thus $L(t)$ is a nondecreasing function on $t \geq 0$. Letting $\delta_{1}$ be small enough in (4.13), then we have $L(0)>0$. Hence

$$
L(t)>0, \text { for } t \geq 0 .
$$

Now set

$$
\theta=\frac{1}{1-\alpha}
$$

Since $\alpha<\alpha_{1}<1$, it is evident that $1<\theta<\frac{1}{1-\alpha_{1}}$. By Young's inequality and Hölder inequality, it follows that

$$
L(t)^{\theta} \leq 2^{\theta-1}\left[H(t)+\left(\delta_{1} \int_{\Omega} u_{t} u d x\right)^{\theta}\right] .
$$

On the other hand, for $p>2$ and using Hölder inequality, we have

$$
\begin{aligned}
\int_{\Omega} u_{t} u d x & \leq\left\|u_{t}\right\|_{2}\|u\|_{2} \\
& \leq c_{7}\left\|u_{t}\right\|_{2}\|u\|_{p},
\end{aligned}
$$

here $c_{7}=(\operatorname{vol}(\Omega))^{\frac{(p-2)}{2 p}}$. And by Young's inequality, we obtain

$$
\begin{aligned}
\left(\int_{\Omega} u_{t} u d x\right)^{\theta} & \leq c_{8}\left\|u_{t}\right\|_{2}^{\theta}\|u\|_{p}^{\theta} \\
& \leq c_{9}\left(\|u\|_{p}^{\theta \beta_{1}}+\left\|u_{t}\right\|_{2}^{\theta \beta_{2}}\right),
\end{aligned}
$$


where $\frac{1}{\beta_{1}}+\frac{1}{\beta_{2}}=1, c_{8}=c_{7}^{\theta}$, and $c_{9}=c_{9}\left(c_{8}, \beta_{1}, \beta_{2}\right)>0$. In particular, we take $\theta_{1} \beta_{2}=2$, i.e. $\beta_{2}=2(1-\alpha)$.

Therefore, for $\alpha$ small enough, the numbers $\beta_{1}$ and $\beta_{2}$ are close to 2. Now choose $\alpha \in\left(0,\left(\alpha_{1}, \frac{1}{2}-\frac{1}{p}\right)\right)$. Note that from (4.10), we see that

$$
\frac{b}{p H(0)}\|u\|_{p}^{p} \geq 1
$$

Thus we obtain

$$
\|u\|_{p}^{\theta \beta_{1}} \leq\left(\frac{b}{p H(0)}\right)^{1-\frac{\theta \beta_{1}}{p}}\|u\|_{p}^{p},
$$

because

$$
\theta \beta_{1}=\frac{2}{1-2 \alpha}<p
$$

Consequently by $(4.18)-(4.20)$, we have

$$
L(t)^{\theta} \leq c_{10}\left[H(t)+\|u\|_{p}^{p}+\left\|u_{t}\right\|_{2}^{2}\right]
$$

here $c_{10}$ is some positive constant. From (4.16) and (4.21), we get

$$
L^{\prime}(t) \geq c_{11} L(t)^{\theta}, t \geq 0
$$

here $c_{11}=\frac{c_{6} \delta_{1}}{c_{10}}$. An integration of $(4.22)$ over $(0, t)$ then yields

$$
L(t) \geq\left(L(0)^{1-\theta}-c_{11}(\theta-1) t\right)^{-\frac{1}{\theta-1}} .
$$

Since $L(0)>0,(4.23)$ shows that $L$ becomes infinite in a finite time $T \leq T^{*}=$ $\frac{L(0)^{1-\theta}}{c_{11}(\theta-1)}$. (II) For $E(0)<0$, we set

$$
H(t)=-E(t),
$$

instead of (4.2). Then, applying the same argunments as in part (I), we have the result.

\section{REFERENCES}

1. R. A. Adams, Sobolev Space, Academic Press, 1975.

2. A. Guesmia, Existence globale et stabilisation interne nonlinéaire d'un systèm de Petrovsky, Bell. Belg. Math. Soc. 5 (1998), 583-594.

3. A. Guesmia, Energy decay for a damped nonlinear coupled system, Journal of Math. Anal. and Applic., 239 (1999), 38-48. 
4. V. Komornik, and S. Kouémou-Patcheu, Well-Posedness and decay estimates for a Petrovsky system with internal damping, Advances in Math. Sci. and Applic., 7 (1997), 245-260.

5. V. Komornik, Well-Posedness and decay estimates for a Petrovsky system by a semigroup approach, Acta Sci. Math. (Szeged), 60 (1995), 451-466.

6. S. A. Messaoudi, Global existence and nonexistence in a system of Petrovsky, Journal of Math. Anal. and Applic., 265 (2002), 296-308.

7. M. Nako, A difference inequality and its application to nonlinear evolution equations, J. Math. Soc. Japan, 30 (1978), 747-762.

8. Y. C. You, Energy decay and exact controllability for the Petrovsky equation in a bounded domain, Advances in Applied Math., 11 (1990), 372-388.

Shun-Tang Wu

General Educational Center,

China University of Technology,

Taipei 116, Taiwan

E-mail: stwu@cute.edu.tw

Long-Yi Tsai

Department of Mathematical Science,

National Chengchi University,

Taipei 116, Taiwan 